\title{
Driving Performance Evaluation Correlated to Age and Visual Acuities Based on VR Technologies
}

\author{
Sooncheon Hwang, ${ }^{1}$ Sunhoon Kim, ${ }^{1}$ and Dongmin Lee $\mathbb{D}^{2}$ \\ ${ }^{1}$ Department of Transportation Engineering, The University of Seoul, 163 Slsiripdae-Ro, Dongdaemun-Gu, Seoul 02504, \\ Republic of Korea \\ ${ }^{2}$ Department of Transportation Engineering \& Department of Smart City, The University of Seoul, 163 Seoulsiripdae-Ro, \\ Dongdaemun-Gu, Seoul 02504, Republic of Korea \\ Correspondence should be addressed to Dongmin Lee; dmlee@uos.ac.kr
}

Received 19 December 2019; Accepted 21 April 2020; Published 1 July 2020

Academic Editor: Inhi Kim

Copyright (@) 2020 Sooncheon Hwang et al. This is an open access article distributed under the Creative Commons Attribution License, which permits unrestricted use, distribution, and reproduction in any medium, provided the original work is properly cited.

\begin{abstract}
There is currently much debate regarding the effectiveness of the driver license system in South Korea, due to the numerous traffic crashes caused by drivers who are suspected of having insufficient physical and mental abilities. Through the present system, it is quite difficult to identify such drivers indirectly through physical tests, such as visual acuity tests, since the correlation of such results with driving performance remains unclear. The objective of this study was to investigate the relationship between driving performance and visual acuities for improving the South Korean driver license system. In this study, two investigations were conducted: static and dynamic visual acuity examinations and driving performance tests based on a virtual reality (VR) system. The driving performance was evaluated with a driving simulator, based on driving behaviors in different experimental scenarios, including daytime and nighttime driving on a rural highway, and unexpected incident situations. Here, we produce statistically significant evidence that reduced visual acuity impairs driving performance, and driving behaviors differ significantly among groups with different vision capabilities, especially dynamic vision. Visual acuities, typically dynamic visual acuity, greatly influenced driving behavior, as measured by the standard deviation of speeds and vehicle LPs, and this was especially notable in curved road segments in daytime experiment. These experimental results revealed that the driving performance of participants with impaired dynamic visual acuity was deficient and unsafe. This confirmed that dynamic visual acuity levels are significant determinants of driving behavior, and they well explain driver performance levels. These findings suggest that the South Korean driver license system should include a test of dynamic visual acuity to create better and safer driving.
\end{abstract}

\section{Introduction}

There are many ways to improve transportation safety, including improving geometric conditions, increasing education, publicizing campaigns, and taking other approaches to improve traffic safety. However, in the context of traffic safety, one of the most important steps is to evaluate whether drivers have adequate capabilities for driving. Driver performance evaluations are critical for identifying individuals who are not qualified to drive because such drivers can seriously affect safety on the road.

Generally, the driver license system is the first stage of preventing traffic crashes, as it evaluates driver performance to identify drivers with inadequate physical abilities. However, there have recently been many arguments regarding the effectiveness of the driver license system in South Korea, due to the many traffic crashes caused by drivers with insufficient physical and mental abilities. The current system in South Korea does not identify such drivers because some of the tests are rather cursory, such as vision and hearing tests. In contrast, many other countries, including Germany, France, Japan, the US, and the UK, have implemented a variety of tests to regularly identify unqualified drivers, including high-level standards for vision tests, viewing angle tests, cognitive ability tests, and other standard medical tests. However, driving performance tests 
cannot be conducted on real roads. It is also quite difficult to evaluate driving performance indirectly through physical tests, such as visual acuity and muscle performance tests, since the correlation of their results with driving performance is unclear. Thus, to improve traffic safety, a new method of accurately evaluating driving performance must be developed.

In this study, driving performance was evaluated through virtual reality (VR) technology to identify unqualified drivers. Recently, VR technology has been used in many areas of transportation engineering, such as driver education, primary driving testing, and driver behavior studies. Such technology has also been used to evaluate driver performance in many studies, due to the difficulty of performing real-time road tests.

The objectives of this study were to determine the relationships between driving performance and physical abilities based on driving simulator experiments and to develop an appropriate method of evaluating driver performance. Physical abilities that are easily measurable and closely related to driver performance were used to develop the evaluation method. Among various physical abilities, the visual acuity levels in different age groups were used to evaluate driving performance, since drivers generally obtain the information needed to safely operate their vehicles through vision, for example, road alignments, road signs, and other driving environments. After visual acuity was examined and driving simulator experiments were conducted, driving behaviors and performance characteristics were analyzed by a statistical modeling process to determine those influencing factors on reaction time which significantly affect traffic crashes.

\section{Literature Review}

It is generally known that drivers get most of the information they need to drive through vision [1], making vision the most important ability for driving. Visual acuity is a measure of the spatial resolution of the visual processing system, and a visual acuity test is an eye exam that checks how well a person sees the details of an object from a specific distance $[2,3]$. There are different types of visual acuities related to driving such as static and dynamic visual acuity.

Visual acuity normally declines with age, as shown in several studies that investigated the relationship between age and visual acuities [4-7]. Another study of vision tests for drivers found that the number of drivers with visual acuity loss was four times greater among those aged 65 years or older than in younger drivers [8]. Those researchers conducted an automated visual inspection on 10,000 drivers and found that only $3 \%$ to $3.5 \%$ of those who were aged 16 to 60 years had impaired vision, whereas $13 \%$ of those who were aged 65 years and over had vision problems.

Insufficient visual acuity may cause poor driving performance, as evidenced by driving violations and traffic crashes [8-12]. Burg investigated the relationship between visual acuity, driving violations, and traffic crashes in California, USA, and found a slight correlation among them [9], and a similar analysis [10] indicated that measures of visual performance, such as static and dynamic visual acuity, correlated significantly with the crash rate of drivers over the age of 54. In another related study of the visual acuity levels (static and dynamic) of 12,400 drivers and their traffic crashes and law violations, it was found that drivers over the age of 66 had a higher risk of crashes [11]. However, those previous studies indirectly analyzed the relationship between a visual acuity and driving performance by tracking the number of violations and traffic crashes. In this study, the influence factors on driving violations and traffic crashes were driving distance, age, gender, and visual acuity (static and dynamic).

Among the various types of visual acuities, dynamic visual acuity is known to significantly affect recognition of traffic ahead and road conditions [13-19]. Hofstetter [14] found a meaningful relationship between dynamic visual acuity and recognition of moving objects, and Long and Kearns [15] also revealed similar results regarding the relationship between traffic sign recognition and dynamic visual acuity. Higgins and Wood [17] tested the effects of visual acuity against measures for driving performance, including gap perception, total driving time, and sign recognition, using a lens designed with different visual acuity levels (ranging from 0.1 to 1.5 ). They observed poor driving performance based on those measures in participants with lower dynamic visual acuity. These results also revealed that vision tests for only static vision acuity (not for dynamic vision acuity) are of limited usefulness in this context. Because the relationship between such tests and driving performance was not really proven statistically, there is still needed to develop effective and reliable methods that can precisely correlate visual acuity and driver performance $[12,20]$.

Several previous studies used a driving simulator to investigate driver behaviors and performance, due to realtime road test limitations such as safety hazards and difficulty in preparing designed situations for experiments [16, 21-24, 25-27]. Only a few studies have used a driving simulator to directly investigate the relationship between visual acuity and driving performance. Wilkins [16] conducted a driving simulator test to measure brake response rates in hazardous situations and found that drivers with better dynamic visual acuity had better risk detection records. In another study on the effects of visual acuity on the driving ability of multiple sclerosis patients, a driving simulator was used to evaluate the subjects' driving performance [28].

There have also been studies recommending the improvement of driver license systems [29, 30]. For example, Owsley and McGwin [21] suggested that it is necessary to measure visual acuity more precisely in the driver licensing system of England, but they failed to find clear evidence that an improved driver licensing system would reduce traffic crashes.

Our reviews of the relevant literature found the following meaningful issues. First, visual acuity, which is highly important for safe driving, normally weakens with age, and this deterioration of vision may cause driving violations and traffic crashes. Thus, declining visual acuity is a critical safety 
problem. Second, dynamic visual acuity is known to significantly affect driving performance, but it is hard to measure it and to prove a statistically relevant relationship between dynamic visual acuity and driving performance. Therefore, more precise methods to measure visual acuities should be developed, and studies to investigate the relationship between visual acuity and driving performance need to be conducted.

\section{Methodology}

In this study, two different investigations were conducted: a visual acuity test and a driving performance test utilizing a VR system. The relationship between driving performance and physical abilities was determined using that VR driving simulator in a series of experiments. Figure 1 illustrates the detailed study flows.

3.1. Visual Acuity Evaluation. For visual acuity evaluations, different types of visual acuities were examined, including static and dynamic visual acuity, because dynamic visual acuity are known to have greater effects on driving performance than static visual acuity [1]. Our visual acuity evaluations were conducted with the Multifunctional Vision Test System developed and verified by the Korea Road Traffic Authority. First, we reanalyzed the visual acuity evaluations results examined by this test system in a previous study [31]. This study was conducted only to evaluate the performance of the vision test system; the data used for verification in that study were used in this study to investigate the reduction in visual acuity performance with aging. Second, the same test system was used to evaluate the visual acuity of the participants in this study, so that subject driving performance could be compared according to visual acuity level. The purpose of this visual acuity test was to evaluate subjects' ability to identify fixed and moving objects. The test results were scored from 0.1 to 1.5 , and subjects with scores greater than 0.5 were judged as having passed the vision test, while the rest were judged as having failed. These criteria were based on the standards of the driving aptitude test used by driving license institutes in South Korea. These thresholds were applied for evaluation of static and dynamic visual acuity.

3.2. Driving Simulator Experiments. We conducted driving simulator experiments to measure various driving behaviors under many different driving conditions. In total, 65 participants ( 35 younger drivers and 30 older drivers, 49 male and 16 female drivers) who drove at least three or four times in every week with an active driver's license were involved in the experiments. Among the younger drivers, there were 11 participants in their twenties, 9 participants in their thirties, 8 participants in their forties, and 7 participants in their fifties; the older drivers were those older than 65 years.

The experiments to evaluate driving performance were conducted in two designed scenarios based on rural highway driving: daytime (scenario 1) and nighttime (scenario 2). Unexpected incident situations, as shown in Figure 2, were

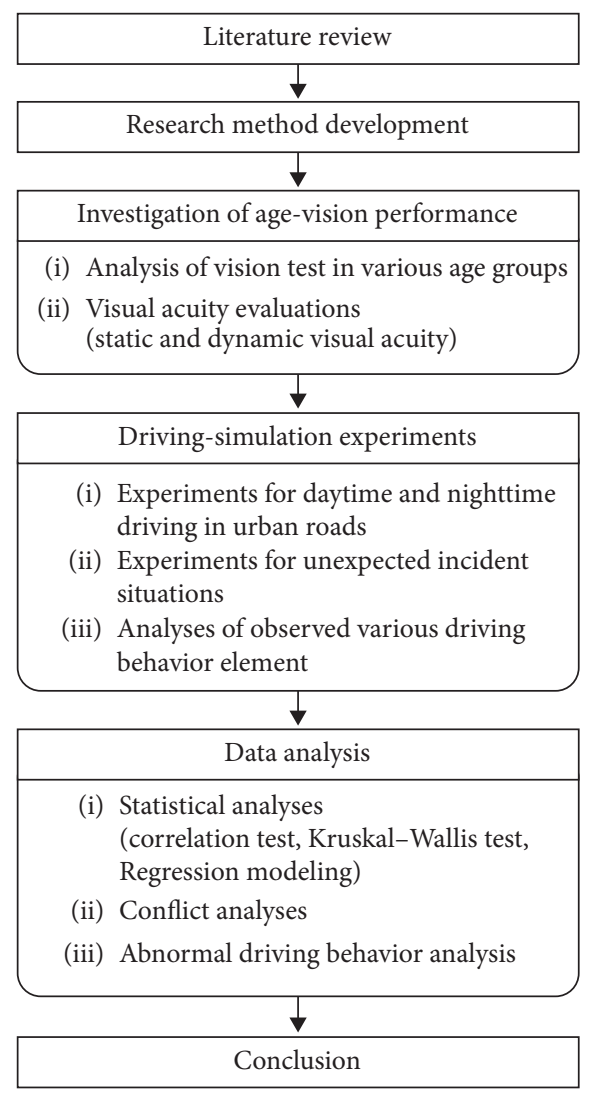

Figure 1: Flowchart of this study.

created in a driving simulator based on VR technology. The unexpected incident \#1 was a dog that suddenly ran into the driving lane, and the driver had to stop to avoid hitting it. In incident \#2, a vehicle in the adjacent lane suddenly cut into the driving lane. In the last incident, a heavy vehicle was approaching from a cross road to an unsignalized intersection.

The visual acuity of each participant was first tested by the vision test system, and then, the driving simulator experiments were conducted. The two different scenarios were presented in random order to each participant to prevent any learning or familiarity effects in the experiments. The VR images in the driving simulator were implemented by the UC-WinRoad program (ver. 12.0) by Forum8 Corp which is in Anyang city, South Korea. Prior to the main test, a predriving test was administered so that participants would become familiar with the driving simulator.

3.3. Statistical Analysis. Correlation analysis is a method to investigate the degree and direction of a relationship between two variables [32], and we conducted it to determine whether visual acuity level influenced driving behavior and performance measures, including variations of speed, braking force, and lateral placement during driving. The Kruskal-Wallis test is a rank-based nonparametric test that can be used to determine whether there is a statistically significant difference between continuous or ordinal dependent variables in two or more groups [33]. We used this 


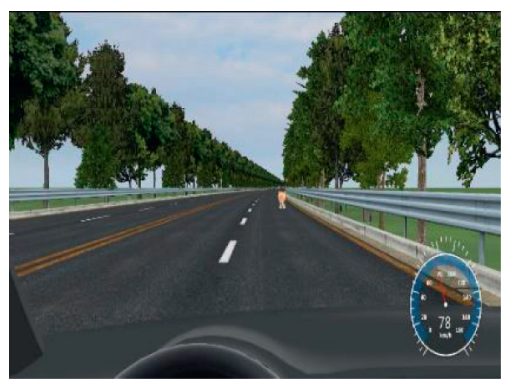

Scenario1-\#1

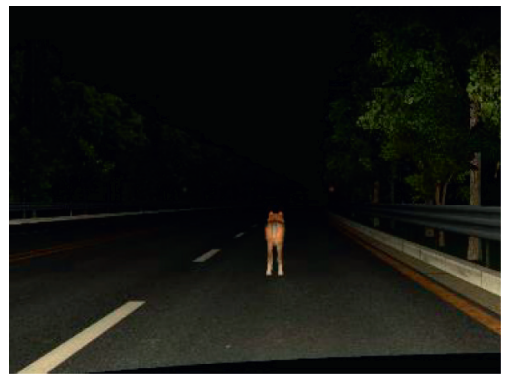

Scenario2-\#1

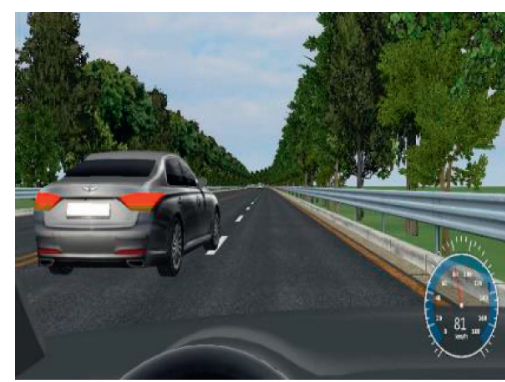

Scenario1-\#2

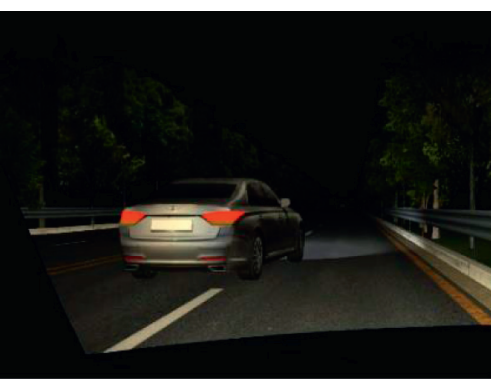

Scenario2-\#2

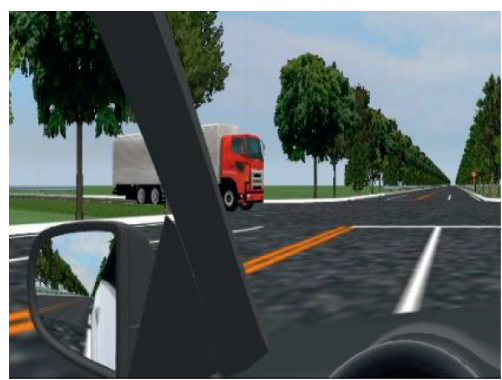

Scenario1-\#3

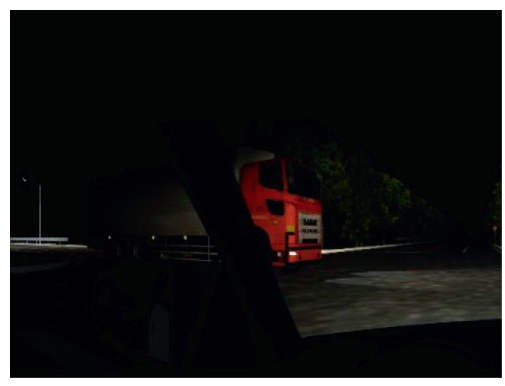

Scenario2-\#3

Figure 2: Images of experimental scenarios.

test to determine whether the occurrence of traffic conflicts in each scenario differed significantly among the groups classified according to visual acuity level. A traffic conflict is an observable event which would result in a crash if drivers do not act appropriately, such as by slowing down, changing lanes, or accelerating to avoid a collision. In this study, conflicts were measured as near-collisions with incident objects, such as a dog, a lane-changing vehicle, or an approaching heavy vehicle, by analysis of videos recorded in the experiment. The number of actual collisions was also included in the number of conflicts here for the purpose of the analysis. Finally, we used regression analysis to determine whether visual acuity levels and age had a significant effect on perception reaction time (PRT) in the unexpected incident situations. This analysis was conducted to determine whether lately perceiving and identifying ahead risky events can increase the probability of traffic crashes and whether the driver's age and visual acuity level are the main influencing factors for those unsafe driving behaviors.

\section{Results}

4.1. Vision Test Results. In agreement with the general knowledge that static visual acuity and dynamic visual acuity decline with aging, our investigation of visual acuity revealed that participants over age 40 had significantly lower visual acuity levels, typically in dynamic visual acuity, than younger participants. In a previous study [31], static and dynamic visual acuities were measured for 276 participants using the same vision measurement device used in this study; we reanalyzed those results to investigate the declination of vision with age. We found large decreases in vision test passing rates among older drivers, especially for dynamic visual acuity, using the Korean standard of static vision acuity in its driver license system, 0.5 points (20/40), as shown in Table 1. Even though the threshold of dynamic visual acuity can be different from that of static visual acuity, we applied the same threshold for dynamic vision.

In our evaluation of these data, $75.4 \%(56.9 \%+18.5 \%)$ of the participants had adequate static visual acuity, as shown in Table 2, while 28 participants $(12+16$ participants $)$ failed the dynamic visual acuity test and 16 participants failed both the dynamic and static visual acuity tests. Such individuals should not drive, considering their insufficient physical performance in terms of dynamic visual ability. As demonstrated here, vision performance typically declines much more rapidly after the age of 50; therefore, driving inspections should be improved for those over the age of 50 .

\subsection{Driving Simulator Experiment Results}

4.2.1. Visual Acuities and Driving Behaviors. In the driving simulator experiments, many participants with lower visual acuity levels drove with higher variations in speed, as can be seen in Figure 3. The data in Figure 3 and Tables 3 and 4 are from the experimental highway segments where no unexpected incidents occurred, meaning these results represent general driving conditions without any effects from incidents. As can be seen in Figures 3(a)-3(c), the standard deviation of driving behavior measures the driving performance, including speed, brake force, and vehicle lateral placement (LP), which were all relatively higher in drivers with lower visual acuities. These trends were slightly more obvious in the dynamic visual acuity results.

Tables 3 and 4 display the correlation results for the daytime (scenario 1) and nighttime (scenario 2) experiments, respectively. These results indicate that in the daytime, visual acuities greatly influenced driving behavior, as measured by the standard deviation of speeds and vehicle 
TABLE 1: Results of the vision test in different age groups.

\begin{tabular}{|c|c|c|c|c|c|}
\hline \multirow[t]{2}{*}{ Age } & \multicolumn{2}{|c|}{$\begin{array}{l}\text { Static visual acuity } \\
\text { (people) }\end{array}$} & \multicolumn{2}{|c|}{$\begin{array}{c}\text { Dynamic visual acuity } \\
\text { (people) }\end{array}$} & \multirow{2}{*}{$\begin{array}{c}\text { Total } \\
\text { (people) }\end{array}$} \\
\hline & Pass (\%) & Fail (\%) & Pass (\%) & Fail (\%) & \\
\hline $20-29$ & $4(100 \%)$ & 0 & $4(100 \%)$ & 0 & 4 \\
\hline $30-39$ & $57(98 \%)$ & $1(2 \%)$ & $57(98 \%)$ & $1(2 \%)$ & 58 \\
\hline $40-49$ & $121(97 \%)$ & $4(3 \%)$ & $115(92 \%)$ & $10(8 \%)$ & 125 \\
\hline $50-59$ & $24(71 \%)$ & $10(29 \%)$ & $14(41 \%)$ & $20(59 \%)$ & 34 \\
\hline $65-69$ & $27(69 \%)$ & $12(31 \%)$ & $11(28 \%)$ & $28(72 \%)$ & 39 \\
\hline Over 70 & $11(69 \%)$ & $5(31 \%)$ & $5(31 \%)$ & $11(69 \%)$ & 16 \\
\hline Total & $244(88 \%)$ & $32(12 \%)$ & $206(75 \%)$ & $70(25 \%)$ & 276 \\
\hline
\end{tabular}

Source: these data are from the Korea Agency for Infrastructure Technology Advancement (2017) [25] to determine whether different age groups passed or failed using the Korean standard for static visual acuity in its driver license system for all visual acuities. The original study merely examined those visual acuities to verify the Multifunctional Vision Test System developed by the study.

TABLE 2: Visual acuity evaluation results.

\begin{tabular}{|c|c|c|c|c|c|c|c|}
\hline \multirow{3}{*}{ No. } & \multicolumn{6}{|c|}{ Groups categorized by visual acuities } & \multirow{3}{*}{ No. of participants (\%) } \\
\hline & \multicolumn{3}{|c|}{ Static } & \multicolumn{3}{|c|}{ Dynamic } & \\
\hline & Result & Mean & $\mathrm{SD}$ & Result & Mean & SD & \\
\hline 1 & Pass & 0.95 & 0.36 & Pass & 0.86 & 0.29 & $37(56.9 \%)$ \\
\hline 2 & Pass & 0.57 & 0.08 & Fail & 0.33 & 0.09 & $12(18.5 \%)$ \\
\hline \multirow[t]{2}{*}{3} & Fail & 0.26 & 0.12 & Fail & 0.21 & 0.09 & $16(24.6 \%)$ \\
\hline & & & Total & & & & $65(100 \%)$ \\
\hline
\end{tabular}

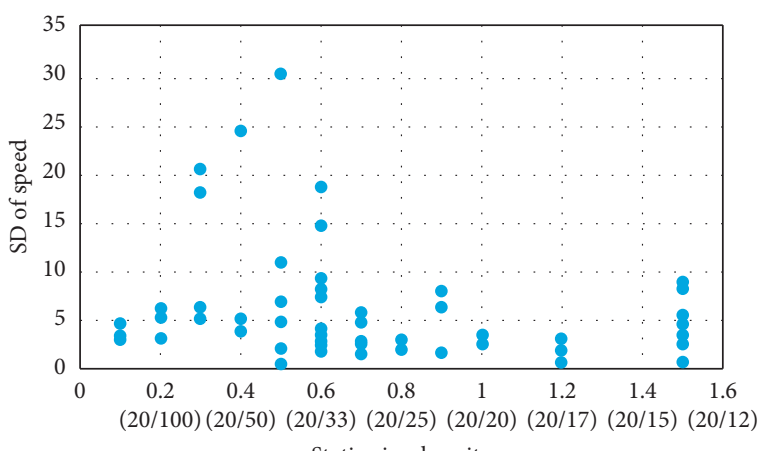

Static visual acuity

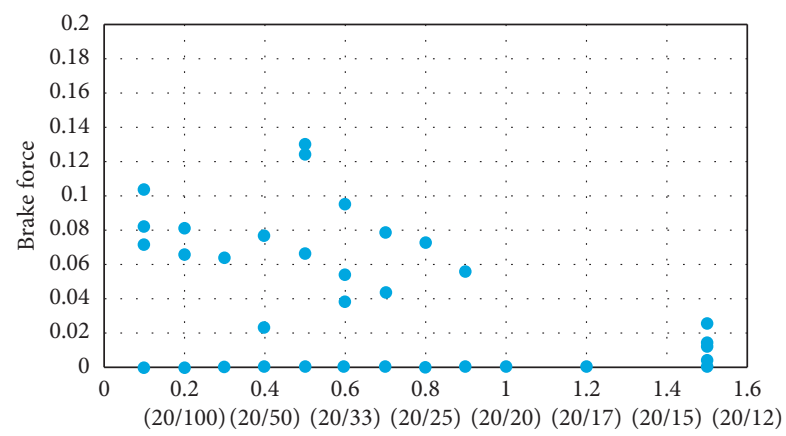

Static visual acuity

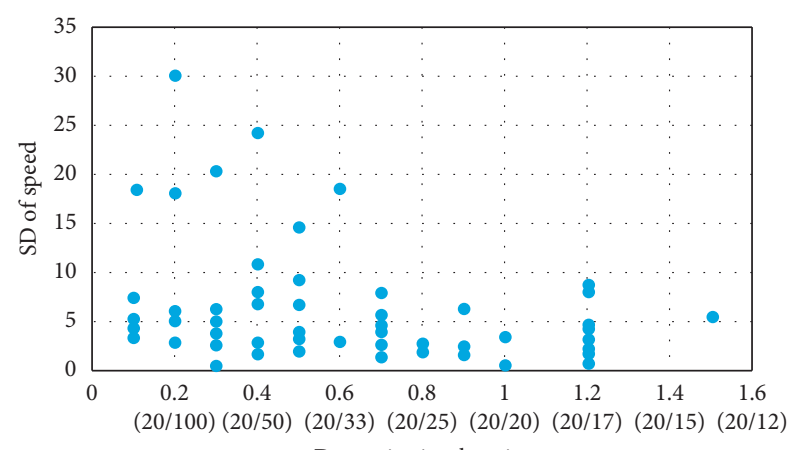

(a)

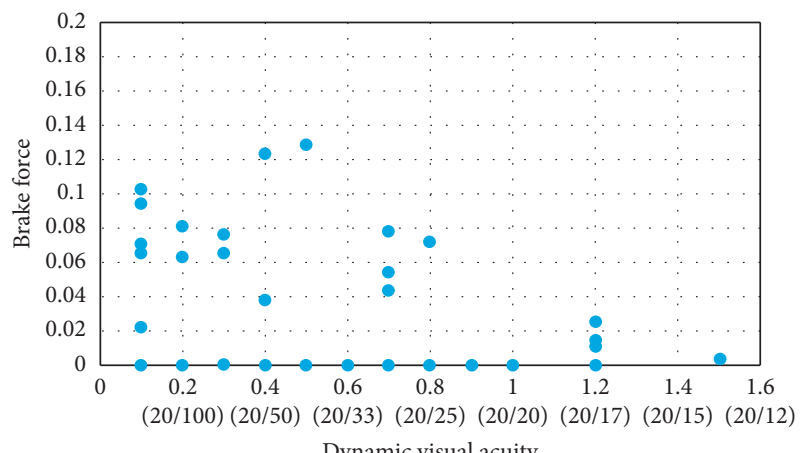

(b)

Figure 3: Continued. 


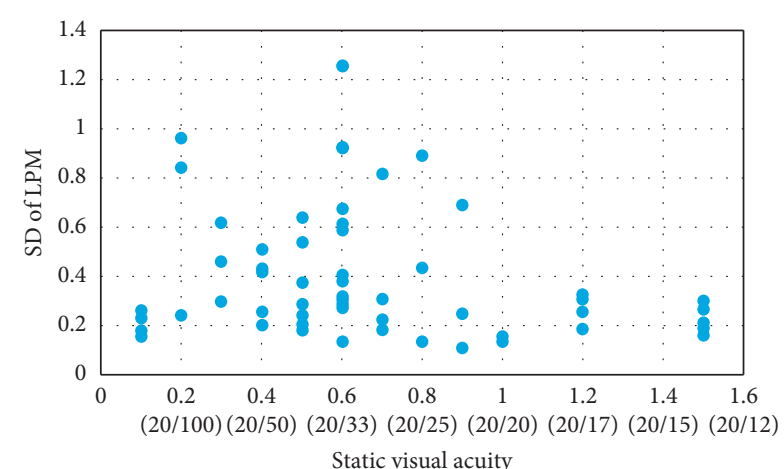

Static visual acuity

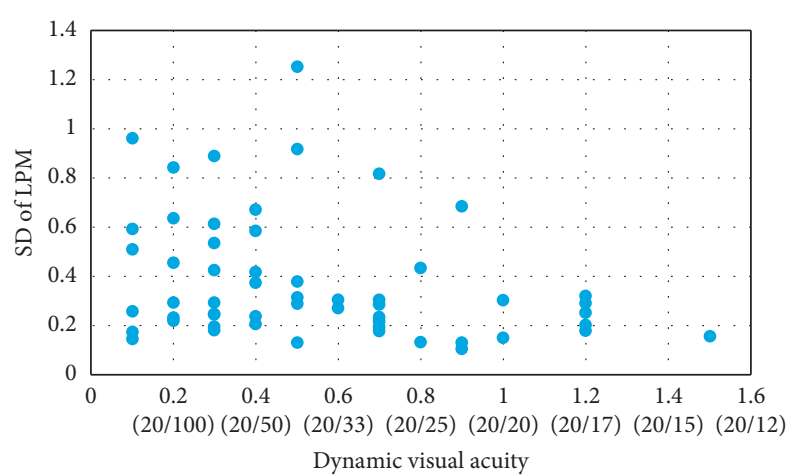

(c)

FIGURE 3: Comparison of driving behaviors according to visual acuity. Comparison of SD of (a) speed, (b) brake force, and (c) lateral placement according to visual acuity.

TABLE 3: Correlation analyses and Kruskal-Wallis tests in scenario 1 (daytime).

\begin{tabular}{|c|c|c|c|c|c|}
\hline & Categories & & SD of running speed & $\mathrm{SD}$ of brake force & SD of LP \\
\hline \multirow{6}{*}{ Correlation test } & \multirow{3}{*}{ Tangent road section } & Static visual acuity & $\begin{array}{c}-0.131 \\
(p=0.299)\end{array}$ & $\begin{array}{c}-0.180 \\
(p=0.150)\end{array}$ & $\begin{array}{c}-0.235 \\
(p=0.059)\end{array}$ \\
\hline & & Dyn & -0.198 & -0.183 & $-0.327^{*}$ \\
\hline & & Dys & $(p=0.114)$ & $(p=0.145)$ & $(p=0.008)$ \\
\hline & \multirow{3}{*}{ Curved road section } & Static visual acuity & $-0.253^{*}$ & -0.127 & $-0.253^{*}$ \\
\hline & & & $(p=0.042)$ & $(p=0.312)$ & $(p=0.042)$ \\
\hline & & Dynamic visual acuity & $\begin{array}{c}-0.356^{*} \\
(p=0.004)\end{array}$ & $\begin{array}{c}-0.183 \\
(p=0.145)\end{array}$ & $\begin{array}{c}-0.352^{*} \\
(p=0.004)\end{array}$ \\
\hline \multirow{2}{*}{ Kruskal-Wallis test } & \multicolumn{2}{|c|}{ Tangent road section } & $\begin{array}{c}\chi^{2}=2.543 \\
(p=0.280)\end{array}$ & $\begin{array}{c}\chi^{2}=0.156 \\
(p=0.925)\end{array}$ & $\begin{array}{l}\chi^{2}=7.223^{*} \\
(p=0.027)\end{array}$ \\
\hline & \multicolumn{2}{|c|}{ Curved road section } & $\chi^{2}=7.586^{*}$ & $\chi^{2}=1.315$ & $\chi^{2}=6.754^{*}$ \\
\hline
\end{tabular}

${ }^{*} p$ value $<0.05$ (statistically significant).

TABLE 4: Correlation analyses and Kruskal-Wallis tests in scenario 2 (nighttime).

\begin{tabular}{|c|c|c|c|c|c|}
\hline & Categories & & SD of running speed & SD of brake force & SD of LP \\
\hline \multirow{6}{*}{ Correlation test } & \multirow{3}{*}{ Tangent road section } & A static visual acuity & -0.036 & 0.079 & -0.065 \\
\hline & & & $(p=0 . / 75)$ & $(p=0.532)$ & $(p=0.606)$ \\
\hline & & A dynamic visual acuity & $\begin{array}{c}-0.069 \\
(p=0.587)\end{array}$ & $\begin{array}{c}0.063 \\
(p=0.620)\end{array}$ & $\begin{array}{c}-0.062 \\
(p=0.626)\end{array}$ \\
\hline & \multirow{3}{*}{ Curved road section } & 4 & -0.210 & -0.149 & -0.160 \\
\hline & & A static visual acuity & $(p=0.093)$ & $(p=0.236)$ & $(p=0.202)$ \\
\hline & & A dynamic visual acuity & $\begin{array}{c}-0.241^{*} \\
(p=0.050)\end{array}$ & $\begin{array}{c}-0.142 \\
(p=0.258)\end{array}$ & $\begin{array}{c}-0.251^{*} \\
(p=0.043)\end{array}$ \\
\hline \multirow{2}{*}{ Kruskal-Wallis test } & \multicolumn{2}{|c|}{ Tangent road section } & $\begin{array}{c}\chi^{2}=1.533 \\
(p=0.465)\end{array}$ & $\begin{array}{c}\chi^{2}=3.065 \\
(p=0.216)\end{array}$ & $\begin{array}{c}\chi^{2}=0.073 \\
(p=0.964)\end{array}$ \\
\hline & \multicolumn{2}{|c|}{ Curved road section } & $\begin{array}{c}\chi^{2}=4.471 \\
(p=0.107)\end{array}$ & $\begin{array}{c}\chi^{2}=0.158 \\
(p=0.924)\end{array}$ & $\begin{aligned} \chi^{2} & =4.049 \\
(p & =0.132)\end{aligned}$ \\
\hline
\end{tabular}

${ }^{*} p$ value $<0.05$ (statistically significant).

LPs, and this was especially notable in curved road segments. Again, these results were more obvious for dynamic visual acuity. Of these driving behavior measures, the standard deviation of vehicle LPs had a stronger correlation with visual acuities, typically dynamic visual acuity. The Kruskal-Wallis test results showed that the differences in the correlations of static and dynamic visual acuities with the standard deviation of vehicle LPs only in the tangent road section, and the standard deviation of speed and vehicle LPs in the curved road section were statistically significant.

Meanwhile, in scenario 2 (nighttime), the correlations between visual acuities and driver behavior measures were less significant than those in the daytime (Table 4). The Kruskal-Wallis test results showed that differences of these correlations of driving behavior measures with static and dynamic visual acuity were not statistically significant in the 
nighttime. This indicates that there might be other influencing factors that were not considered in this study, such as nighttime visual acuity.

4.2.2. Visual Acuity and Driving Conflicts. Traffic conflicts while driving in unexpected incident situations in the daytime were used for comparing the groups divided according to the determined visual acuity level. Table 5 displays the average number of conflicts for each group. The largest number of conflicts (3.3) was recorded for those who passed the static acuity test but failed the dynamic acuity test. Fewer conflicts (2.1) were observed for those who passed all the acuity tests than for those who failed all of the tests (2.2). Participants who failed the dynamic vision test had more conflicts during the experiments, as can be seen in Table 5.

These conflict results showed that the drivers with insufficient dynamic visual acuity (even if they had acceptable static visual acuity) were more frequently involved in traffic accidents. This confirms that dynamic visual acuity levels strongly affect driving behavior and help to determine the driving performance level. These findings suggest that the driver license system in South Korea should include tests of dynamic visual acuity to create safer driving conditions.

4.2.3. Analysis of the Effects of Visual Acuities on Reaction Time. When a driver is slow to perceive and identify upcoming dangerous situations, the risk of a collision increases. For this reason, analyzing the effects of visual acuities on the time needed for perceiving and identifying can be meaningful. However, because it is generally quite difficult to measure exact time for perceiving and identifying approaching objects in the VR simulator experiment, we used the entire perception reaction time (PRT) instead of just time to perceive and identify ahead the object. The reaction time used in this study was derived from the following equation:

$$
\text { measured PRT }=T_{1}-T_{2},
$$

Measured PRT ( $T_{1}$ and $T_{2}$ were measured using raw data related to driving behaviors that the driving simulator automatically produced.): time difference between time $\left(T_{1}\right)$ when a driver starts to step on the brake and time $\left(T_{2}\right)$ when the hazard obstacle appears ahead.

The results showed a significant correlation between the measured PRT and actual conflicts for incidents 1 and 3 in both the daytime and nighttime experiments, as shown in Table 6. However, results for incident 2 in the daytime and nighttime experiments were not significant. This might be explained that incidents 1 and 3 occurred some distance ahead and were completely unexpected, but incident 2 , in which a vehicle in the adjacent lane suddenly cut into the driving lane, occurred in front of participants' very eyes. Therefore, incident 2 might have been relatively less affected by the driver's visual acuity. These results demonstrate that PRT has a significant relationship with conflicts and possibly with traffic accidents.
TABLE 5: Comparison of driving conflicts according to visual acuity performance.

\begin{tabular}{lccc}
\hline \multicolumn{2}{c}{$\begin{array}{c}\text { Groups by determined visual } \\
\text { acuities }\end{array}$} & Average conflicts of groups \\
Group no. & Static & Dynamic & \\
\hline 1 & Pass & Pass & 2.1 \\
2 & Pass & Fail & 3.3 \\
3 & Fail & Fail & 2.2 \\
\hline
\end{tabular}

A regression analysis was conducted to investigate the significant visual acuities that affected the measured PRT (Table 7). Dynamic visual acuity had statistically significant effects in both daytime and nighttime situations, but static visual acuity was not statistically significant. The PRT decreased as the dynamic visual acuity decreased, as the coefficient of the dynamic visual acuity was negative. There was little difference in the coefficient values between daytime and nighttime conditions. This result confirms that dynamic visual acuity, which measures how accurately a moving object is observed, is an important driver aptitude in both daytime and nighttime conditions.

4.2.4. Ability to Cope with Incidents according to Visual Acuities. Generally, drivers with vision problems do not cope well with sudden incidents, due the association of poor vision with impaired cognitive capacity to respond quickly and accurately. The experiments in this study confirmed this general tendency, as shown in Figure 4. Old drivers and nonold drivers who passed the static and dynamic visual acuity levels (Group 1) had similar speed and brake force patterns (Figure 4(a)), but those who passed the static visual acuity test but failed the dynamic visual acuity test (Group 2) had different speed and brake force patterns. For example, to cope with incident \#3, non-old drivers started to brake and reduced speed at the appropriate time, but old drivers started to brake and reduced speed too late (Figure 4(b)). As mentioned previously, about $70 \%$ of the old participants and about $30 \%$ of the non-old participants in this experiment failed in the dynamic vision test. In sudden incident events, the worst behaviors were observed in participants with insufficient dynamic vision acuity (about $10 \%$ of the drivers), including sudden harsh braking due to late identification of the incident. However, this difference was smaller in participants who failed both static and dynamic vision tests (Group 3) as can be seen in Figure 4(c). It might be because drivers with poor static vision performance usually more concentrate on ahead conditions in driving and are ready to cope with them.

Figure 5 shows two examples of those extreme cases. The first case involved a 50-year-old female participant with 20 years of driving experience; she had driven five times per week but failed both the static and dynamic visual acuity tests. The other case was an older male driver with 30 years of driving experience who had driven every day. He failed the dynamic visual acuity tests, although he passed the static visual acuity test. Neither subject identified sudden events fast enough to successfully respond. When the woman 
TABLE 6: Correlation analyses between reaction time and conflicts in three incidents.

\begin{tabular}{lccc}
\hline \multicolumn{2}{l}{ Categories } & & Conflicts \\
& & Scenario 1 (daytime) & Scenario 2 (nighttime) \\
\hline & Incident \#1 & $0.462^{*}$ & $0.564^{*}$ \\
Reaction time & (dog runs into road) & $(p<0.001)$ & $(p<0.001)$ \\
& Incident \#2 & 0.063 & $0.331^{*}$ \\
& (car in adjacent lane veers) & $(p=0.666)$ & $(p=0.020)$ \\
(heavy vehicle approaches) & $0.653^{*}$ & $0.632^{*}$ & $(p<0.001)$ \\
\hline
\end{tabular}

${ }^{*} p$ value $<0.05$ (statistically significant).

TABLE 7: Regression models for conflicts using visual acuities.

\begin{tabular}{lcccr}
\hline \multirow{2}{*}{ Model } & Variable & Unstandardized coefficients & Std. error & \multirow{2}{*}{ Sig. } \\
& & B & 0.417 & 0.000 \\
\multirow{2}{*}{ Daytime } & Constant & $5.827^{*}$ & 0.638 & 13.986 \\
& Dynamic visual acuity & $-1.560^{*}$ & -2.447 & 0.018 \\
\hline \multirow{2}{*}{ Nighttime } & Constant & $2.644^{*}$ & 0.421 & 6.279 \\
& Dynamic visual acuity & $-1.658^{*}$ & 0.645 & -2.572 \\
\hline
\end{tabular}

${ }^{*} p$ value $<0.05$ (statistically significant).
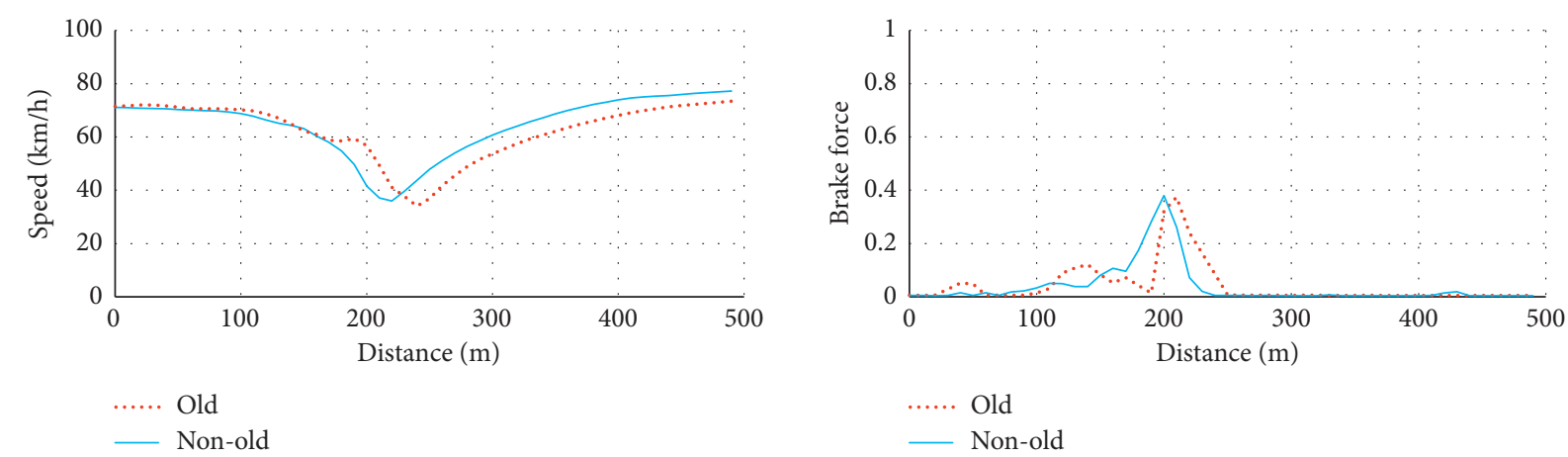

(a)
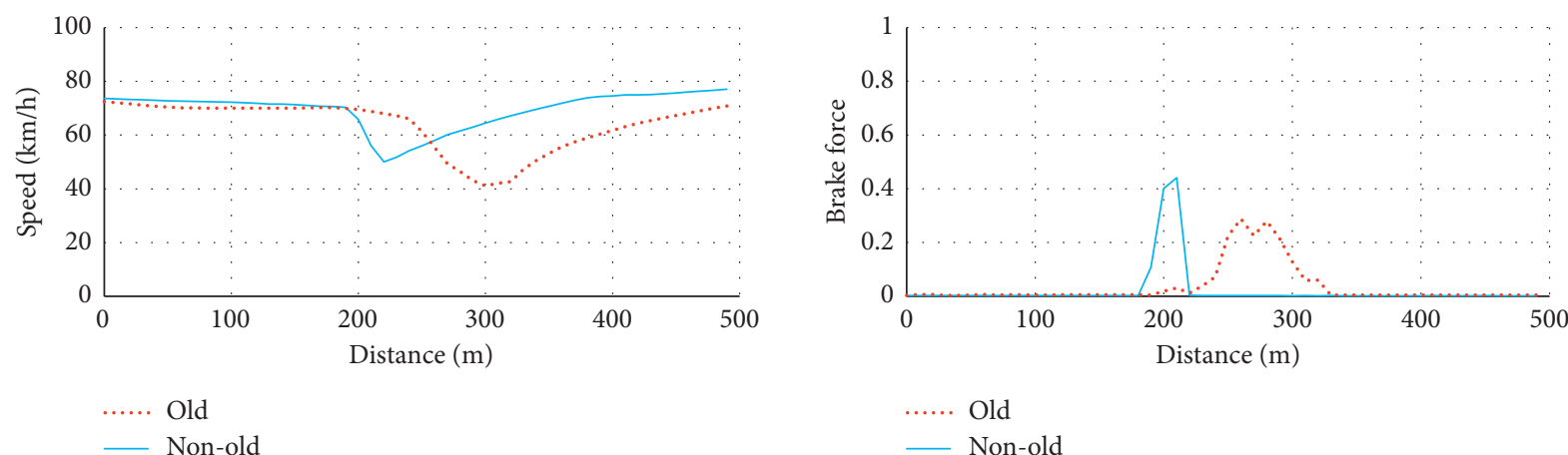

(b)

Figure 4: Continued. 


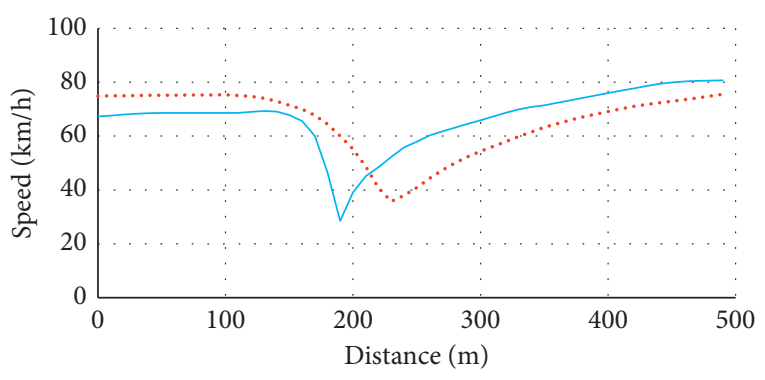

$\begin{array}{ll}\ldots . . . & \text { Old } \\ & \text { Non-old }\end{array}$

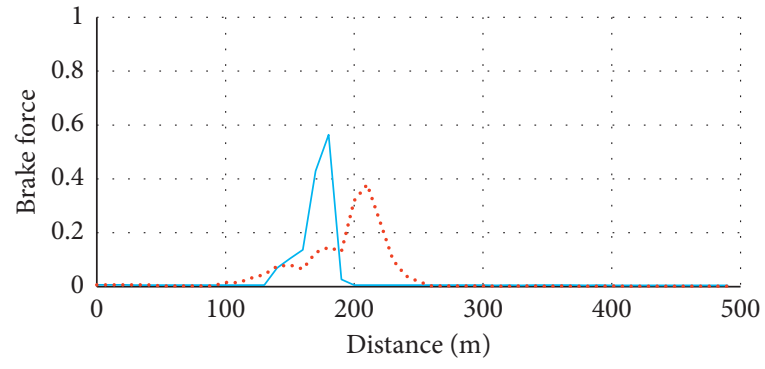

.... Old

— Non-old

(c)

FIGURE 4: Comparison of driving behaviors according to age for incident \#3 in the daytime experiment. (a) Group 1 (passed both static and dynamic vision tests). (b) Group 2 (passed the static vision test but failed the dynamic vision test). (c) Group 3 (failed both static and dynamic vision tests).

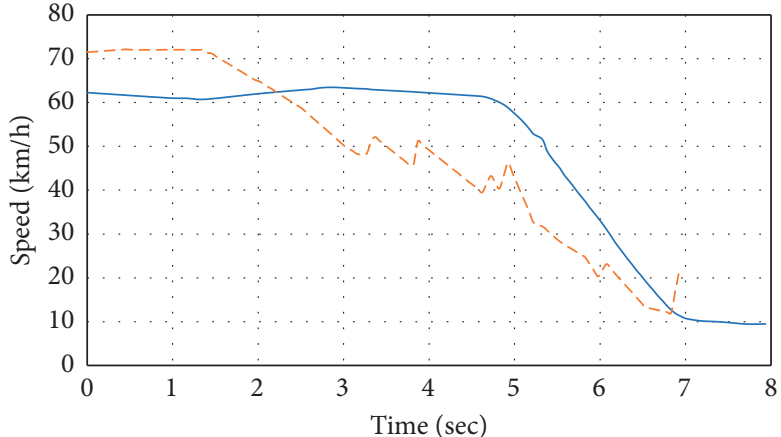

— Results of the participant

-- Average of nonelderly participants

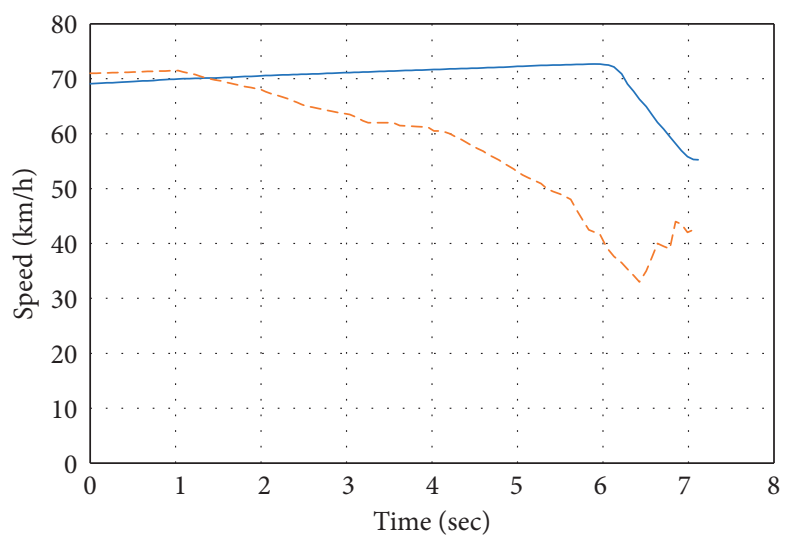

— Results of the participant

--- Average of elderly participants

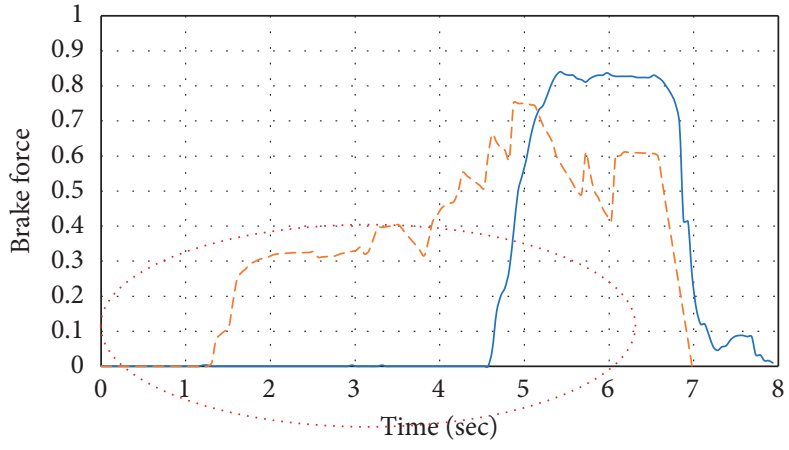

(a)

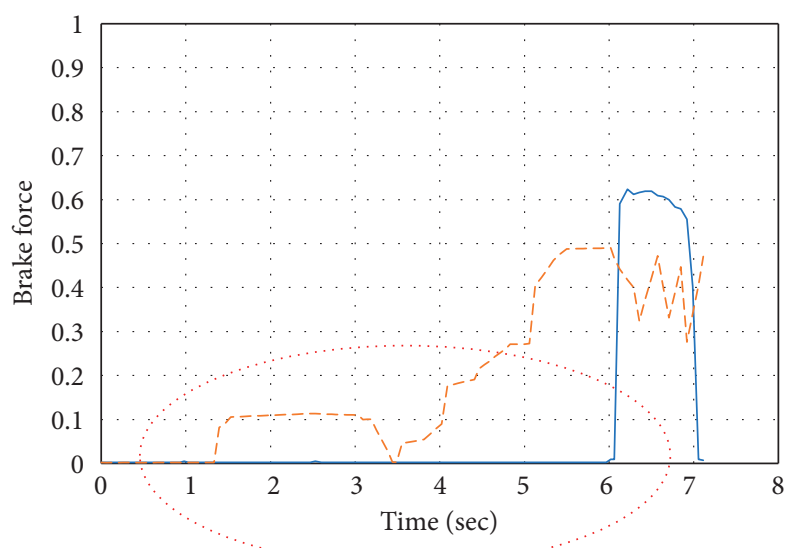

(b)

Figure 5: Cases of two problematic participants in the daytime experiment with incident \#3. (a) The problematic female participant. (b) The problematic male participant.

identified a sudden event too late, she braked suddenly with great force, demonstrating how in real-life situations, severe crashes can be caused by low visual acuity. Figure 5 illustrates these participants' behaviors.

\section{Conclusions}

For the sake of traffic safety, it is of vital importance to determine whether drivers have adequate capabilities for 
driving and to keep unqualified drivers off the roads. Therefore, driver performance evaluations are essential for identifying those not qualified to drive. However, there is currently much debate regarding the effectiveness of the driver license system in South Korea, due to the many traffic crashes caused by drivers with insufficient physical and mental abilities [34-39]. It is also quite difficult to assess driving performance indirectly through physical tests, such as visual acuity and muscle performance tests, because the correlation of those results with driving performance is to date unclear. Thus, we conducted a driving performance evaluation to improve traffic safety based on VR technology.

In the driving simulator experiments, many participants with lower visual acuity levels drove with greater variations in speed, failed to brake appropriately when confronted with sudden incidents, and failed to avoid crashes. Here, we produce statistically significant evidence that reduced visual acuity impairs driving performance, and through designed VR experiments, we demonstrate that the driving performance of participants with insufficient dynamic visual acuity can cause unsafe situations. We also found that dynamic acuity has a great deal of influence on driving performance in both the daytime and nighttime, and it can be an effective criterion for driver qualification. These findings suggest that the driver license system in South Korea should consider including dynamic visual acuity testing for better and safer driving.

However, there were some limitations to this study. The number of participants was only 65 , not enough for analyzing the trends of declining visual acuity. Also, driving in virtual scenarios is different from driving on real roads. Nevertheless, this pioneering study shows how dynamic visual acuity can explain driving performance; more scientific studies are needed to prove that dynamic visual acuity is a reliable screening factor which does not change easily according to external circumstances or conditions. Also, further studies with larger sample sizes are needed to analyze more precisely the trends of visual acuity with aging, as well as more detailed physical and mental criteria for driving, such as hand-eye coordination, with the goal of finding clearer correlations between age and driving performance. It is necessary to test the driving performance of these same 65 participants on real roads to assess the similarity with the findings of this study. And finally, as noted earlier, this study applied the same threshold for both static and dynamic visual acuities, even though they can be different. To address this study limitation, more precise criteria and thresholds for dynamic visual acuity should be determined through more scientific driving performance studies.

\section{Data Availability}

The data used to support the findings of this study are available from the corresponding author upon request.

\section{Conflicts of Interest}

The authors declare that there are no conflicts of interest regarding the publication of this paper.

\section{Acknowledgments}

This work was partially supported by the 2019 Research Fund of the University of Seoul for Dongmin Lee. Also, this research was supported by the Basic Science Research Program through the National Research Foundation of Korea (NRF) funded by the Ministry of Education (2018R1D1A1B07049554) for Sooncheon Hwang.

\section{References}

[1] D. Shinar, Traffic Safety and Human Behavior, Emerald Publishing Limited, Bingley, UBingley, UK, 2nd edition, 2017.

[2] https://en.wikipedia.org/wiki/visual_acuity.

[3] C. Owsley, K. Ball, M. E. Sloane, D. L. Roenker, and J. R. Bruni, "Visual/cognitive correlates of vehicle accidents in older drivers," Psychology and Aging, vol. 6, no. 3, pp. 403415, 1991.

[4] J. L. Keltner and C. A. Johnson, "Mass visual field screening in a driving population," Ophthalmology, vol. 87, no. 8, pp. 785-792, Aug. 1980.

[5] G. McGwin, V. Chapman, and C. Owsley, "Visual risk factors for driving difficulty among older drivers," Accident Analysis and Prevention, vol. 32, no. 6, pp. 735-744, 2000.

[6] C. Owsley, "Aging and vision," Vision Research, vol. 51, no. 13, pp. 1610-1622, 2011.

[7] D. R. Ragland, W. A. Satariano, and K. E. MacLeod, "Reasons given by older people for limitation or avoidance of driving," The Gerontologist, vol. 44, no. 2, pp. 237-244, 2004.

[8] C. A. Johnson and J. L. Keltner, "Incidence of visual field loss in 20,000 eyes and its relationship to driving performance," Archives of Ophthalmology, vol. 101, no. 3, pp. 371-375, 1983.

[9] A. Burg, "Vision and driving: a report on research," Human Factors, vol. 13, pp. 79-87, 1971.

[10] B. L. Hills and A. Burg, "A reanalysis of California driver vision data general findings," TRRL Report 768, U.K. Transport and Road Research Laboratory, Crowthorne, England, 1977.

[11] L. E. Decina, L. Staplin, and A. S. Spiegel, Correcting Unaware Vision Impaired Drivers, No. 730009, Pennsylvania Department of Transportation, Harrisburg, PA, USA, 1990.

[12] S. Subzwari and E. Desapriya, "Vision screening of older drivers for preventing road traffic injuries and fatalities," Cochrane Database of Systematic Reviews, 2006.

[13] A. Burg, "Lateral visual field as related to age and sex," Journal of Applied Psychology, vol. 52, no. 1, pp. 10-15, 1968.

[14] H. W. Hofstetter, "Visual acuity and highway crashes," Journal of the American Optometric Association, vol. 47, pp. 887-893, 1976.

[15] G. M. Long and D. F. Kearns, "Visibility of text and icon highway signs under dynamic viewing conditions," Human Factors: The Journal of the Human Factors and Ergonomics Society, vol. 38, no. 4, pp. 690-701, 1996.

[16] L. Wilkins, R. Gray, J. Gaska, and M. Winterbottom, "Motion perception and driving: predicting performance through testing and shortening braking reaction times through training," Investigative Opthalmology and Visual Science, vol. 54, no. 13, p. 8364, 2013.

[17] K. E. Higgins and J. M. Wood, "Predicting components of closed road driving performance from vision tests," Optometry and Vision Science, vol. 82, no. 8, pp. 647-656, 2005.

[18] H. Wang, X. Mo, Y. Wang, R. Liu, P. Qiu, and J. Dai, "Assessing Chinese coach drivers' fitness to drive: the 
development of a toolkit based on cognition measurements," Accident Analysis and Prevention, vol. 95, pp. 395-404, 2016.

[19] T. Abe, K. Fujii, J. Seol et al., "Driving frequency associated with deficits in lower extremity function, dynamic vision, and physical activity in Japanese older adults," Journal of Transport and Health, vol. 9, pp. 282-287, 2018.

[20] D. Shinar and F. Schieber, "Visual requirements for safety and mobility of older drivers," Human Factors: The Journal of the Human Factors and Ergonomics Society, vol. 33, no. 5, pp. 507-519, 1991.

[21] C. Owsley and G. McGwin, "Vision impairment and driving," Survey of Ophthalmology, vol. 43, no. 6, pp. 535-550, 1999.

[22] A. K. Pradhan, K. R. Hammel, R. DeRamus, A. Pollatsek, D. A. Noyce, and D. L. Fisher, "Using eye movements to evaluate effects of driver age on risk perception in a driving simulator," Human Factors: The Journal of the Human Factors and Ergonomics Society, vol. 47, no. 4, pp. 840-852, 2005.

[23] H. L. Lew, J. H. Poole, E. H. Lee, D. L. Jaffe, H.-C. Huang, and E. Brodd, "Predictive validity of driving-simulator assessments following traumatic brain injury: a preliminary study," Brain Injury, vol. 19, no. 3, pp. 177-188, 2005.

[24] J. K. Muguro, M. Sasaki, and K. Matsushita, "Evaluating hazard response behavior of a driver using physiological signals and car-handling indicators in a simulated driving environment," Journal of Transportation Technologies, vol. 9, no. 4, pp. 439-449, 2019.

[25] J. Lengenfelder, M. T. Schultheis, T. Al-Shihabi, R. Mourant, and J. DeLuca, "Divided attention and driving," Journal of Head Trauma Rehabilitation, vol. 17, no. 1, pp. 26-37, 2002.

[26] D. Lee and J. Lim, "Driver behavior Analyses of $2+1$ roads based on a driving simulation experiment," KSCE Journal of Civil Engineering, vol. 23, no. 3, pp. 1351-1359, 2019.

[27] S. C. Hwang, S. H. Kim, and D. M. Lee, "Driving behavior on hard-shoulder lanes in tunnels using a driving simulator," International Journal of Highway Engineering, vol. 21, no. 3, pp. 87-96, 2019.

[28] S. Krasniuk, S. Classen, and S. A. Morrow, "Relationships among vision, visual attention, and fitness to drive in adults with multiple sclerosis," American Journal of Occupational Therapy, vol. 71, no. 4, Article ID 7111500009p1, 2017.

[29] S. C. Kang and S. W. Lee, "an analysis of older drivers' riskiness using driving simulator and driving aptitude test," Traffic Science Institute; Research of Traffic Safety, vol. 32, pp. 5-14, 2013.

[30] D. C. Grabowski, "Elderly licensure laws and motor vehicle fatalities," JAMA, vol. 291, no. 23, p. 2840, 2004.

[31] Korea Agency for Infrastructure Technology Advancement, Development of Driving Fitness Test Based on Virtual Reality, Korea Agency for Infrastructure Technology Advancement, Anyang, Gyeonggi-do, Republic of Korea, 2017.

[32] J. Cohen, P. Cohen, S. G. West, and L. S. Aiken, Applied Multiple Regression/Correlation Analysis for the Behavioral Sciences, Erlbaum, Mahwah, NJ, USA, 3rd edition, 2003.

[33] D. Othayoth and K. V. Krishna Rao, "Factors influencing level of service for motorized vehicles at signalized intersection under mixed traffic condition," Transportation in Developing Economies, vol. 3, no. 2, 2017.

[34] S. J. Kang, S. H. Choi, B. H. Lee, J. C. Kwon, D. L. Na, and S. H. Han, "The reliability and validity of the Korean instrumental activities of daily living," Journal of the Korean Neurological Association, vol. 20, no. 1, pp. 8-14, 2002.

[35] Korea Centers for Disease Control and Prevention, The Risk of Driving in Dementia Patients, Korea Centers for Disease Control and Prevention, Cheongju-si, South Korea, 2009.
[36] S. J. Park, S. C. Lee, and H. R. Jang, "The influence of driving situational adaptability and chronic disease on driving behavior of elderly drivers," Korean Journal of Psychological and Social Issues, vol. 14, no. 2, pp. 1-19, 2008.

[37] S. Y. Park, Y. H. Kim, and H. C. Shin, "Selection framework of driving simulator scenarios for driver education based on traffic accident data analysis," Journal of Transport Research, vol. 23, no. 4, pp. 15-33, 2016.

[38] J. Charlton, S. Koppel, M. Odell et al., Influence of Chronic Illness on Crash Involvement of Motor Vehicle Drivers, Report No. 300, Monash University Accident Research Centre, Clayton, Victoria, Australia, 2nd edition, 2010.

[39] S. W. van Landingham, C. Hochberg, R. W. Massof, E. Chan, D. S. Friedman, and P. Y. Ramulu, "Driving patterns in older adults with glaucoma," BMC Ophthalmology, vol. 13, no. 1, 2013. 